\title{
Diagnostic value of CD163 in cutaneous spindle cell lesions
}

Background: The histologic diagnosis of atypical fibroxanthoma (AFX) can sometimes be challenging. No specific marker exists to confirm the diagnosis other than excluding other entities. CD163 has been shown to have great specificity for tumors of monocyte/histiocyte lineage. In this study, we evaluated the diagnostic utility of CD163 in diagnosing AFX and in identifying skin lesions with histiocytic/ dendritic derivation.

Methods: A total of 157 cases, including 14 AFXs, 5 spindle cell squamous cell carcinomas (SCGs), and 7 spindle cell/desmoplastic melanomas, along with other cutaneous spindle cell and histiocytic/ fibrohistiocytic lesions, were stained with CD163.

Results: CD163 was expressed in 11 of $14(79 \%)$ AFXs, with moderate to strong intensity. No staining was observed in cases of spindle cell SCG $(0 / 5)$ and dermatofibrosarcoma protuberans $(0 / 10)$. Rare spindle cell/desmoplastic melanomas (2/7) and cutaneous leiomyosarcomas (1/5) demonstrated positive staining. CD163 reactivity was seen in 24 of 29 of benign fibrous histiocytomas (BFHs), including 8 of 8 celular fibrous histiocytomas and 6 of 9 epithelioid cell histiocytomas. The majority of cutaneous histiocytic lesions, including juvenile xanthogranuloma, Langerhans cell histiocytosis and RosaiDorfman disease, were positive for CD163.

Conclusion: CD163 is a useful adjunct in distinguishing AFX from other malignant cutaneous spindle cell tumors and offers improved specificity in identifying cutaneous histiocytic/dendritic lesions.

Pouryazdanparast P, Yu L, Cutlan JE, Olsen SH, Fullen DR, Ma L. Diagnostic value of CD163 in cutaneous spindle cell lesions.

J Cutan Pathol 2009; 36: 859-864. (C) 2008 John Wiley \& Sons A/S.
Pedram Pouryazdanparast ${ }^{\mathbf{1}}$, Limin Yu' ${ }^{1}$, Jonathan E. Cutlan', Stephen H. Olsen ${ }^{1,2}$, Douglas R. Fullen ${ }^{1,2}$ and Linglei $\mathrm{Ma}^{1,2}$

${ }^{1}$ Department of Pathology and ${ }^{2}$ Department of Dermatology, University of Michigan, Ann Arbor, MI, USA
Linglei Ma, MD, PhD, Department of Pathology, Dermatopathology Division, University of Michigan, M3260, Medical Sciences 1, 1301 Catherine Road, Ann Arbor, Ml 48109-0602, USA

Tel: +17347644579

Fax: +17347644690

e-mail: lingleim@umich.edu

Accepted for publication September 11, 2008
The morphologic diagnosis of cutaneous malignant spindle cell tumors, such as atypical fibroxanthoma (AFX), spindle cell squamous cell carcinoma (SCG), and spindle cell/desmoplastic melanoma, may sometimes pose a diagnostic dilemma. Immunohistochemical studies are often required to confirm or exclude a diagnosis. Occasionally, dermatofibrosarcoma protuberans (DFSP) and cutaneous leiomyosarcoma may be included in the differential diagnosis. In general, AFX is a diagnostic exclusion based on negative immunoreactivity for cytokeratin and S-100 protein. Conventional histiocytic markers, such as CD68, have been widely used to confirm the diagnosis of AFX.
However, it has low specificity and may stain a wide variety of neoplasms, including melanomas and carcinomas. ${ }^{1,2}$ In addition, some poorly differentiated SCCs may show a lack of cytokeratin expression, ${ }^{3,4}$ and occasional spindle cell/desmoplastic melanomas may fail to react with S-100 protein. ${ }^{5}$

As a result, new markers are being sought after to diagnose AFX with more certainty. S100A6 is expressed in up to $80 \%$ of AFXs as well as in $33 \%$ of melanomas., CD99 immunoreactivity was reported in $73 \%$ of $\mathrm{AFXs}$, with rare cases of melanoma being positive, suggesting its value in diagnosing AFX. ${ }^{8}$ In a recent study, procollagen- 1 was found to 


\section{Pouryazdanparast et al.}

be positive in $87 \%$ of $\mathrm{AFXs}$, in contrast to one third of desmoplastic melanomas and spindle cell SCGs being reactive. ${ }^{9}$ CD10 has been recently recommended in the diagnosis of AFX, with positivity observed in 94-100\% of tumors. ${ }^{10,11}$ However, $33 \%$ of spindle cell/desmoplastic melanomas and one half of spindle cell SCGs also showed weak and patchy staining. ${ }^{11}$

The hemoglobin-haptoglobin scavenger receptor protein $(\mathrm{CD} 163 / \mathrm{HbSR})$ is a monocyte-/macrophagerestricted transmembrane glycoprotein of the scavenger receptor cysteine-rich family. ${ }^{12-14}$ In addition to its function as an endocytic receptor for iron homeostasis, CD163 is also involved in anti-inflammatory processes. ${ }^{12}$ CD163 antigen is strongly expressed on tissue macrophages, while weak expression is commonly seen on peripheral blood monocytes. ${ }^{1,2}$ Recently, a CD163 monoclonal antibody (clone 10D6) became commercially available for use in paraffin-embedded tissue samples. It was shown to label not only mature monocytes and macrophages but also neoplastic cells in tumors of monocyte/ histiocyte lineage, such as acute myelomonocytic leukemia. ${ }^{2}$ When compared to other conventional histiocytic markers, CD163 demonstrates greater tissue specificity with no reactivity for carcinomas or melanomas. ${ }^{1,2}$ In a small series, CD163 expression was found in one of three cases of AFX. ${ }^{2}$ More recently, CD163 was shown to be a useful marker in distinguishing between cellular fibrous histiocytomas and DFSPs, given its higher expression in the former lesion. ${ }^{15}$

To date, the utilization of CD163 in a full range of cutaneous spindle cell tumors and cutaneous histiocytic/fibrohistiocytic lesions has not been systemically examined. In this study, we proposed that CD163 may be a useful marker in the diagnosis of certain cutaneous spindle cell tumors, given its limited range of expression and tissue specificity. We also tested the diagnostic utility of CD163 in identifying skin lesions with histiocytic/dendritic origin.

\section{Materials and methods}

Case selection and tissue microarray construction

The study was approved by the Institutional Review Board at the University of Michigan Health System and was carried out in accordance with the guidelines for human subject research. As listed in Table 1, a spectrum of cutaneous spindle cell tumors as well as benign histiocytic/fibrohistiocytic lesions and their mimickers was identified through a retrospective search of the pathology database from the Department of Pathology at the University of Michigan. Hematoxylin and eosin-stained sections, together with relevant immunohistochemical stains, were

Table 1. CD163 expression in cutaneous spindle cell tumors and fibrohistiocytic lesions

\begin{tabular}{|c|c|c|c|c|c|}
\hline \multirow[b]{2}{*}{ Diagnosis (TMA and non-TMA) } & \multirow[b]{2}{*}{$\mathrm{n}$} & \multicolumn{3}{|c|}{ Percentage of CD163-positive cells } & \multirow[b]{2}{*}{ Overall positivity } \\
\hline & & $<10$ (negative) & $10-50$ & $>50$ & \\
\hline Atypical fibroxanthoma & 14 & 3 & 5 & 6 & 11/14 (79\%) \\
\hline Spindle cell squamous cell carcinoma & 5 & 5 & 0 & 0 & $0 / 5(0 \%)$ \\
\hline Desmoplastic melanoma & 7 & 5 & 1 & 1 & $2 / 7(29 \%)$ \\
\hline Leiomyosarcoma & 5 & 4 & 1 & 0 & $1 / 5(20 \%)$ \\
\hline Dermatofibrosarcoma protuberans & 10 & 10 & 0 & 0 & $0 / 10(0 \%)$ \\
\hline Dermatofibroma (see variants below) & 29 & 5 & 16 & 8 & $24 / 29(83 \%)$ \\
\hline Conventional & 8 & 2 & 5 & 1 & $6 / 8(75 \%)$ \\
\hline Cellular variant & 8 & 0 & 6 & 2 & $8 / 8(100 \%)$ \\
\hline Hemosiderotic variant & 2 & 0 & 1 & 1 & $2 / 2(100 \%)$ \\
\hline Lipidized variant & 1 & 0 & 0 & 1 & $1 / 1(100 \%)$ \\
\hline Sclerotic variant & 1 & 0 & 1 & 0 & $1 / 1(100 \%)$ \\
\hline Epithelioid cell histiocytoma & 9 & 3 & 3 & 3 & $6 / 9(67 \%)$ \\
\hline Leiomyoma & 8 & 8 & 0 & 0 & $0 / 8(0 \%)$ \\
\hline Dermatomyofibroma & 5 & 5 & 0 & 0 & $0 / 5(0 \%)$ \\
\hline Neurofibroma & 10 & 9 & 1 & 0 & $1 / 10(10 \%)$ \\
\hline Cellular neurothekeoma & 2 & 2 & 0 & 0 & $0 / 2(0 \%)$ \\
\hline Superficial acral fibromyxoma & 2 & 2 & 0 & 0 & $0 / 2(0 \%)$ \\
\hline Juvenile xanthogranuloma & 9 & 0 & 0 & 9 & $9 / 9(100 \%)$ \\
\hline Reticulohistiocytoma & 2 & 0 & 0 & 2 & $2 / 2(100 \%)$ \\
\hline Xanthoma & 4 & 0 & 3 & 1 & $4 / 4(100 \%)$ \\
\hline Langerhans cell histiocytosis & 3 & 0 & 2 & 1 & $3 / 3(100 \%)$ \\
\hline Rosai-Dorfman & 2 & 0 & 0 & 2 & $2 / 2(100 \%)$ \\
\hline Granuloma annulare & 7 & 0 & 5 & 2 & $7 / 7(100 \%)$ \\
\hline Sarcoidosis & 7 & 1 & 4 & 2 & $6 / 7(86 \%)$ \\
\hline Epithelioid sarcoma & 3 & 3 & 0 & 0 & $0 / 3(0 \%)$ \\
\hline Fibrous papule & 4 & 1 & 2 & 1 & $3 / 4(75 \%)$ \\
\hline Mast cell disease & 6 & 6 & 0 & 0 & $0 / 6(0 \%)$ \\
\hline Kaposi's sarcoma & 5 & 5 & 0 & 0 & $0 / 5(0 \%)$ \\
\hline Malignant fibrous histiocytoma & 8 & 2 & 2 & 4 & $6 / 8(75 \%)$ \\
\hline
\end{tabular}

TMA, tissue microarray. 
Diagnostic value of CD163

reviewed by two dermatopathologists (L. M. and D. R. F.) to confirm the original diagnoses. In particular, all AFX cases included in the study were diagnosed based on the histologic features and on the exclusion of other entities through immunohistochemical study.

Formalin-fixed and paraffin-embedded tissue blocks were retrieved, and the diagnostic area of interest was selected for the cylinder core. The tissue microarrays (TMAs) were constructed using an ATA100 Tissue Arrayer (Millipore, Temecula, CA, USA) as previously described. ${ }^{16} \mathrm{~A}$ total of 157 cases were included in this study. Most of cutaneous spindle cell tumors and histiocytic/fibrohistiocytic lesions (130 cases) were constructed on TMA. Nineteen TMA cases that lacked sufficient tissue for evaluation were excluded from the study. For lesions unsuitable for TMA (46 cases), such as sarcoidosis and granuloma annulare, conventional unstained slides were used.

\section{Immunohistochemistry}

The unstained slides from the TMA and conventional paraffin blocks were stained with CD163 antibody. Briefly, 4- $\mu \mathrm{m}$-thick unstained sections were pretreated with Ventana CG1 Solution ( $\mathrm{pH}$ 8.0) for antigen retrieval and were incubated with NCL-CD163 monoclonal antibody (clone 10D6, 1:50 dilution; Vision Biosystems, Norwell, MA, USA) using a Ventana Benchmark XT system (Ventana Medical Systems, Tucson, AZ, USA). The UltraVision System and a 3,3'-diaminobenzidine chromagen were used for detection.

Cytoplasmic and membranous staining was considered positive for CD163. According to the percentage of positive lesional cells, the staining was semiquantitatively recorded as $<10 \%$ (negative), $10-50 \%$ or $>50 \%$. The staining intensity was graded as weak, moderate or strong. Two pathologists (L. M. and P. P.) independently scored the CD163 staining for all cases.

\section{Statistical analysis}

Statistical analysis was carried out using SAS 8.2 software (SAS Institute Inc., Cary, NC, USA). The Fisher's exact test was used to assess the difference in CD163 expression between AFX and other malignant cutaneous spindle cell tumors. A p-value $<0.05$ was considered statistically significant.

\section{Results}

In normal skin, CD163 labeled the dermal dendritic cells (data not shown). A cytoplasmic and membranous staining in at least $10 \%$ lesional cells was considered positive for GD163. When entrapped intralesional dendritic cells or macrophages were stained, it was not considered as true positivity for lesional cells. The results of CD163 expression in a number of skin lesions are summarized in Table 1 .

Among malignant cutaneous spindle cell tumors, CD163 was diffusely expressed in most AFXs (11/14; $79 \%$ ), with all positive cases demonstrating moderate to strong intensity (Fig. 1A). In contrast, none of the spindle cell SCG $(0 / 5)$ and DFSP $(0 / 10)$ reacted with CD163 (Fig. 1B, G). Most spindle cell/desmoplastic melanomas were negative for CD163 (Fig. 1D), with the exception of two cases $(2 / 7 ; 29 \%)$ showing moderate staining. One leiomyosarcoma (1/5) demonstrated focal positivity with moderate intensity, while the rest were non-reactive. The CD163 staining pattern in AFX was significantly different from other that in malignant cutaneous spindle cell tumors, including spindle cell SCC, spindle cell/desmoplastic melanoma, DFSP and leiomyosarcoma $(\mathrm{p}<0.05$ for each). Interestingly, most cases $(6 / 8 ; 75 \%)$ of malignant fibrous histiocytoma (MFH) demonstrated moderate to strong staining for CD163, similar to that seen in AFXs.

As shown in Table 1, CD163 expression was observed in most cases $(24 / 29 ; 83 \%$ ) of benign fibrous histiocytoma $(\mathrm{BFH})$ regardless of the tumor subtypes (Fig. 2A). Among 9 epithelioid cell histiocytomas tested, the majority $(6 / 9 ; 67 \%)$ reacted positively. Of all positive BFHs, 20 of 24 displayed moderate to strong staining (Fig. 2B). We noted that diffuse and strong CD163 expression was more frequently seen in hemosiderotic $\mathrm{BFH}$, lipidized $\mathrm{BFH}$ and epithelioid cell histiocytoma, whereas sclerotic variant or paucicellular areas in conventional BFH often had focal and weak labeling. No CD163 reactivity was observed in dermatomyofibromas (0/5), leiomyomas (0/8), cellular neurothekeomas $(0 / 2)$ or superficial acral fibromyxomas $(0 / 2)$ (Fig. 2C). Except for one case (1/10, $10 \%)$, neurofibromas were not stained with CD163. Most cases of fibrous papule (3/4) were positive, with two showing weak staining intensity.

Among cutaneous histiocytic lesions, strong CD163 positivity was observed in all cases of juvenile xanthogranuloma (9/9), reticulohistiocytoma (2/2), Langerhans cell histiocytosis (3/3), Rosai-Dorfman disease (2/2) and granuloma annulare (7/7) (Fig. 2D). The majority of sarcoidosis $(6 / 7 ; 86 \%)$ had strong staining. All xanthomas (4/4) were positive for CD163, with most (3/4) showing weak intensity. No CD163 reactivity was observed in mimicking lesions, including epithelioid sarcoma (0/3), Kaposi's sarcoma (0/5) and mastocytosis (0/6).

\section{Discussion}

CD163 is a hemoglobin scavenger receptor and functions as an endocytic receptor-scavenging 


\section{Pouryazdanparast et al.}
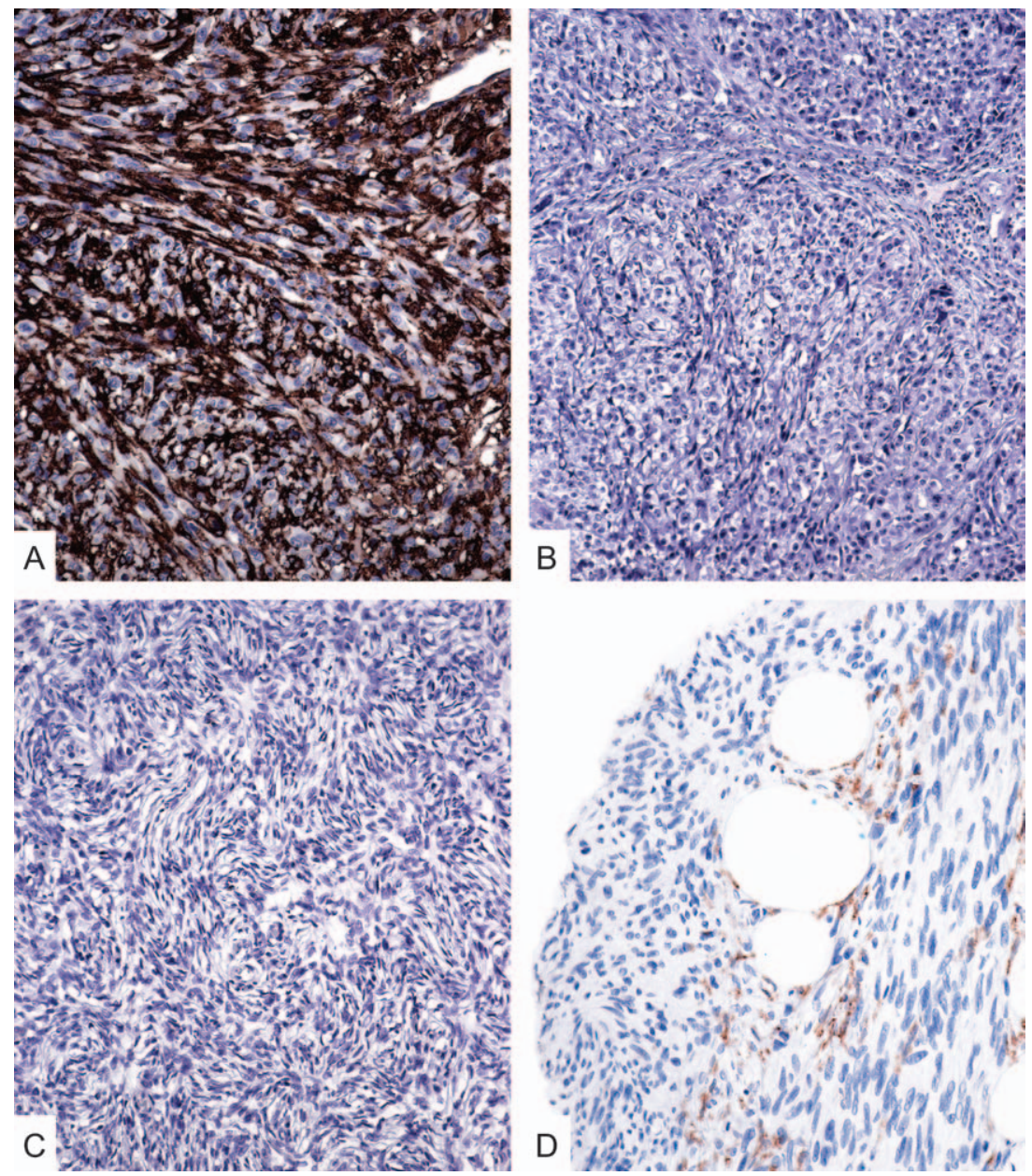

Fig. 1. CD163 staining in cutaneous malignant spindle cell tumors. A) Atypical fibroxanthoma with diffuse and strong CD163 staining in tumor cells. There is a lack of CD163 reactivity in spindle cell squamous cell carcinoma $(\mathrm{B})$, dermatofibrosarcoma protuberans $(\mathrm{C})$ and spindle cell/desmoplastic melanoma (D) (original magnification, $\times 200)$.

haptoglobin-hemoglobin complexes. ${ }^{12}$ It has a greater tissue specificity for cells with monocyte/macrophage differentiation. In skin, CD163 is typically expressed in dermal macrophages and dendritic cells. ${ }^{1,13,14}$ Recently, CD163 expression has been found in tumors with monocytic and histiocytic derivation, such as acute myeloid leukemia with monocytic differentiation and Rosai-Dorfman disease. In the same study, none of the carcinomas or melanomas were reactive. ${ }^{1,2}$ Of interest, Nguyen et al. showed that a subset of AFX cases (1/3) were CD163 positive, while all melanomas $(0 / 22)$ were non-reactive. ${ }^{2}$ However, their cohort was small, and other malignant cutaneous spindle cell tumors, such as spindle cell SCC, were not investigated. Although their 22 melanomas were reported to be negative for CD163, there was no detailed information regarding the morphologic subtypes of these melanomas. In the current study, we examined the expression of CD163 in more cases of AFX in comparison to its histologic mimickers.
In our series, the majority $(11 / 14)$ of AFXs displayed CD163 positivity with moderate to strong intensity, while no cases of spindle cell SCG or DFSP showed CD163 expression. Although a few cases of spindle cell/desmoplastic melanoma (2/7) and cutaneous leiomyosarcoma $(1 / 5)$ showed some staining, the difference in CD163 expression between AFX and other malignant cutaneous spindle cell tumors was statistically significant $(\mathrm{p}<0.05)$. Our findings suggest that CD163 is a useful marker in separating AFX from other entities when used together with the conventional immunohistochemical panel for malignant cutaneous spindle cell tumors. Regarding the occasional cases of desmoplastic melanoma and leiomyosarcoma showing CD163 positivity, we suspect that the positive staining is probably because of antigen cross-reactivity.

The diagnosis of AFX is, by definition, dependent on the use of a wide panel of immunohistochemical markers. It is invariably negative for keratins, S-100 protein and desmin. In the past, there have been efforts toward establishing a confirmatory stain for 

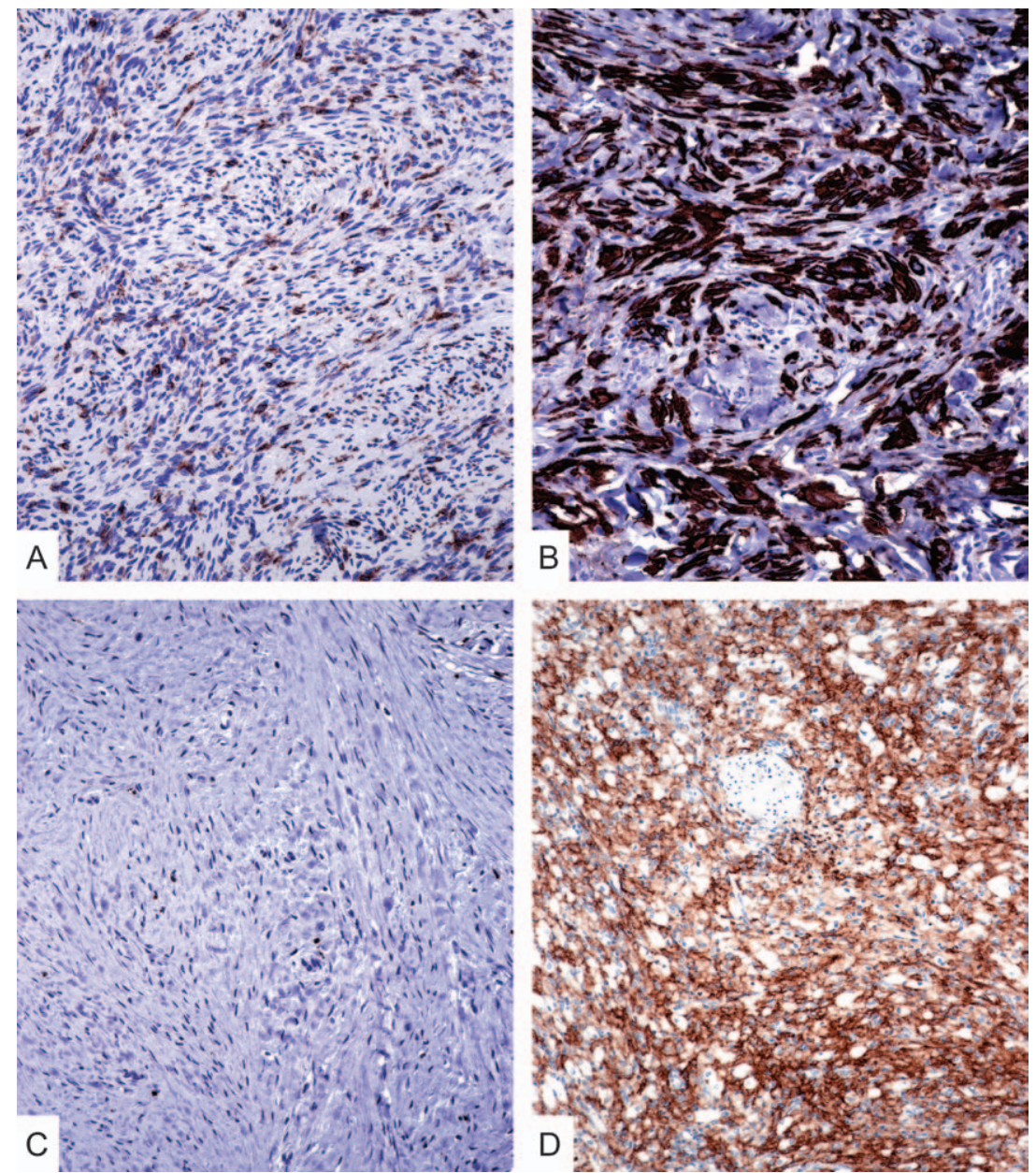

Fig. 2. GD163 expression in benign cutaneous histiocytic/fibrohistiocytic lesions. A) Conventional dermatofibroma with moderate staining intensity in tumor cells. B) Epithelioid cell histiocytoma showing diffuse and strong CD163 reactivity. C) Dermatomyofibroma displaying negative staining. D) Juvenile xanthogranuloma with diffuse labeling in lesional cells (original magnification, ×200).

AFX. Markers such as CD68, lysozyme, alpha-1antitrypsin and alpha-1-antichymotrypsin are commonly expressed in AFX but have low specificity. ${ }^{17,18}$ A number of recent studies have offered several potentially useful diagnostic markers, including CD99, procollagen- 1 and CD10.8,10,11 The fact that CD163 expression was nearly restricted in AFX makes it another useful adjunct in the diagnosis of AFX. However, because of the presence of CD163 reactivity in a small subset of spindle cell/desmoplastic melanomas and cutaneous leiomyosarcomas, special caution should be taken to avoid diagnosing AFX based exclusively on CD163 positivity. In addition, it should be made aware that some entrapped CD163-positive dendritic cells/macrophages may be seen within the tumor, and these cells should not be interpreted as positive tumor cells. As the CD163 positivity was observed in both $\mathrm{AFX}$ and $\mathrm{MFH}$, it is not helpful in separating the two entities.

Earlier work has shown that a subset of $\mathrm{BFH}$ reacted with GD163. ${ }^{2}$ More recently, Sachdev and Sundram ${ }^{15}$ found that CD163 was expressed in the majority of $\mathrm{BFH}(89 \%)$ and cellular fibrous histiocytoma $(100 \%)$, but only in a small number of DFSP $(17 \%, 3 / 18)$, indicating its potential utility in the differential diagnosis of cellular BFH and DFSP. Our data confirm and expand their results. We found that CD163 is expressed in 83\% (24/29) of BFH, including the cellular variant. None of our DFSPs $(0 / 10)$ were labeled with CD163. Both histiocytic and spindle cell components were labeled positively, similar to that reported in a previous study. ${ }^{15}$ Intriguingly, we observed that $67 \%$ of epithelioid cell histiocytomas reacted diffusely and intensely with CD163. Nguyen et al. have made a similar observation in a single case. ${ }^{6}$ Inconsistent with our findings, Miettinen and Fetsch ${ }^{19}$ stated that, in their experience (unpublished observation), the main cellular component of epithelioid cell histiocytoma is negative for CD163. Histologically, epithelioid cell histiocytoma resembles lesions comprising epithelioid cells, such as Spitz nevi and cellular neurothekeoma. ${ }^{20,21}$ Although factor XIIIa is usually an adequate marker to help with the diagnosis, potential pitfalls exist in that occasional cases of 


\section{Pouryazdanparast et al.}

epithelioid cell histiocytoma may have focal S-100 protein positivity and/or lack the expression of factor XIIIa. ${ }^{20}$ Our data suggest that CD163 can aid with the diagnosis in this settings.

In this study, we also tested a wide spectrum of cutaneous histiocytic/fibrohistiocytic lesions. CD163 reactivity was not observed in cases of dermatomyofibroma, cellular neurothekeoma or superficial acral fibromyxoma, a novel finding not previously reported. In concordance with earlier studies, ${ }^{1,2,19}$ we found CD163 expression in all lesions of juvenile xanthogranuloma, reticulohistiocytoma, RosaiDorfman disease and Langerhans cell histiocytosis. As expected, most histiocytic lesions, such as granuloma annulare and sarcoidosis, reacted positively with CD163. In contrast, epithelioid sarcoma, mastocytosis and Kaposi's sarcoma were CD163 negative. Our work further supports the notion that CD163 is a relatively specific marker for lesions of histiocyte/ dendrocyte derivation and expands the utility of CD163 in diagnosing skin lesions. ${ }^{1,2}$

The potential histogenesis of AFX has been controversial. Some investigators have proposed that AFX may represent a reactive process, while others contend that it is a fibrohistiocytic neoplasm, closely related to $\mathrm{MFH}$. It is variously regarded as being of myofibroblastic, fibrohistiocytic and fibroblastic differentiation. $^{22,23}$ The expression of CD163 in AFX suggests that it may, in part, be related to histiocytic/ dendritic lineage. Alternatively, AFX lesions may have acquired an antigenic determinant of histiocyte/ dendrocyte through partial differentiation. Nonetheless, continued investigation is necessary to unravel the true histogenesis of AFX.

In summary, CD163 appears to be a useful marker in identifying cutaneous lesions of histiocytic/dendritic lineage. Our data suggest that CD163 may be a useful adjunct to the immunohistochemical panel of AFX. It appears helpful in separating $\mathrm{BFH}$ from DFSP and particularly in assisting with the diagnosis of epithelioid cell histiocytoma. Future research with larger study populations is necessary to establish the utility of CD163 in diagnosing malignant cutaneous spindle cell tumors.

\section{References}

1. Lau SK, Chu PG, Weiss LM. CD163: a specific marker of macrophages in paraffin-embedded tissue samples. Am J Clin Pathol 2004; 122: 794.

2. Nguyen TT, Schwartz EJ, West RB, Warnke RA, Arber DA, Natkunam Y. Expression of CD163 (hemoglobin scavenger receptor) in normal tissues, lymphomas, carcinomas, and sarcomas is largely restricted to the monocyte/macrophage lineage. Am J Surg Pathol 2005; 29: 617.

3. Sigel JE, Skacel M, Bergfeld WF, House NS, Rabkin MS, Goldblum JR. The utility of cytokeratin 5/6 in the recognition of cutaneous spindle cell squamous cell carcinoma. J Cutan Pathol 2001; 28: 520.

4. Prieto VG, Lugo J, McNutt NS. Intermediate- and lowmolecular-weight keratin detection with the monoclonal antibody MNF116. An immunohistochemical study on 232 paraffin-embedded cutaneous lesions. J Cutan Pathol 1996; 23: 234.

5. Blessing K, Sanders DS, Grant JJ. Comparison of immunohistochemical staining of the novel antibody melan-A with S100 protein and HMB-45 in malignant melanoma and melanoma variants. Histopathology 1998; 32: 139.

6. Ribe A, McNutt NS. S100A6 protein expression is different in Spitz nevi and melanomas. Mod Pathol 2003; 16: 505.

7. Fullen DR, Reed JA, Finnerty B, McNutt NS. S100A6 expression in fibrohistiocytic lesions. J Cutan Pathol 2001; 28: 229.

8. Monteagudo G, Calduch L, Navarro S, Joan-Figueroa A, Llombart-Bosch A. CD99 immunoreactivity in atypical fibroxanthoma: a common feature of diagnostic value. Am J Clin Pathol 2002; 117: 126.

9. Jensen K, Wilkinson B, Wines N, Kossard S. Procollagen 1 expression in atypical fibroxanthoma and other tumors. J Cutan Pathol 2004; 31: 57.

10. Weedon D, Williamson R, Mirza B. CD10, a useful marker for atypical fibroxanthomas. Am J Dermatopathol 2005; 27: 181.

11. Hultgren TL, DiMaio DJ. Immunohistochemical staining of CD10 in atypical fibroxanthomas. J Cutan Pathol 2007; 34: 415.

12. Kristiansen M, Graversen JH, Jacobsen C, et al. Identification of the haemoglobin scavenger receptor. Nature 2001; 409: 198.

13. Ochoa MT, Loncaric A, Krutzik SR, Becker TC, Modlin RL. "Dermal dendritic cells" comprise two distinct populations: CD1(+) dendritic cells and CD209(+) macrophages. J Invest Dermatol 2008; 128: 2225.

14. Zaba LC, Fuentes-Duculan J, Steinman RM, Krueger JG, Lowes MA. Normal human dermis contains distinct populations of CD11c+BDCA-1+ dendritic cells and CD163+FXIIIA+ macrophages. J Clin Invest 2007; 117: 2517.

15. Sachdev R, Sundram U. Expression of CD163 in dermatofibroma, cellular fibrous histiocytoma, and dermatofibrosarcoma protuberans: comparison with CD68, CD34, and Factor XIIIa. J Gutan Pathol 2006; 33: 353.

16. Nocito A, Kononen J, Kallioniemi OP, Sauter G. Tissue microarrays (TMAs) for high-throughput molecular pathology research. Int J Cancer 2001; 94: 1.

17. Pernick NL, DaSilva M, Gangi MD, Crissman J, Adsay V. "Histiocytic markers" in melanoma. Mod Pathol 1999; 12: 1072.

18. McHugh M, Miettinen M. KPl (CD68): its limited specificity for histiocytic tumors. Appl Immunohistochem 1994; 2: 186 .

19. Miettinen M, Fetsch JF. Reticulohistiocytoma (solitary epithelioid histiocytoma): a clinicopathologic and immunohistochemical study of 44 cases. Am J Surg Pathol 2006; 30: 521.

20. Glusac EJ, McNiff JM. Epithelioid cell histiocytoma: a simulant of vascular and melanocytic neoplasms. Am J Dermatopathol 1999; $21: 1$.

21. Zelger BG, Steiner H, Kutzner H, Maier H, Zelger B. Cellular 'neurothekeoma': an epithelioid variant of dermatofibroma? Histopathology 1998; 32: 414.

22. Longacre TA, Smoller BR, Rouse RV. Atypical fibroxanthoma. Multiple immunohistologic profiles. Am J Surg Pathol 1993; 17: 1199.

23. Ma CK, Zarbo RJ, Gown AM. Immunohistochemical characterization of atypical fibroxanthoma and dermatofibrosarcoma protuberans. Am J Clin Pathol 1992; 97: 478. 